\author{
Brenton Stewart \\ Louisiana State University, Baton Rouge, LA, USA
}

Jessie J. Walker

Jackson State University, Jackson, MS, USA

\title{
Twitter and the Lack of a Participatory Culture in American College Libraries (Paper)
}

\begin{abstract}
This paper presents results of a social media analysis on the use of Twitter by academic libraries at majority-minority colleges and universities in the United States.
\end{abstract}

\section{Résumé:}

Academic libraries have readily adopted social networking into their day-to-day information services and some researchers posit social media "can be an effective method of student outreach" (emphasis added) (Dickson \& Holley, 2010). However, do we really know if libraries are connecting with users via social platforms? Leveraging social media under the banner of outreach is the public performance of a rhetorical refrain known as Library 2.0. A derivative of Web 2.0, which describes the second iteration of the Internet, emphasizing increased interaction and collaboration, Library 2.0 describes an ambiguous assortment of activities that include some form of user interaction with digital information communication technologies that can support a participatory library culture (Deodato, 2014). The problem with Library 2.0 and adoption of technologies like Twitter is the relative absence of empirical evidence demonstrating these tools are in fact connecting with library users. John Bushman argues the library profession engages in an "uncritical hype of technologies" that "celebrate[s]" while offering little if any “evaluation" (Bushman, 2003 161). Of the few studies examining Twitter in academic libraries, we found they were situated in research-intensive universities, ignoring not only smaller colleges and university libraries but also, libraries serving a large number of minority students. This analysis of Historically Black Colleges and Universities libraries, in the United States, presents an opportunity to observe both occurrences. Our research asks the broad question: Are users engaging with library generated content on Twitter?

\section{Literature Review}

Previous research in this domain is overwhelmingly situated at large and elite universities, and does not quantify user engagement at a micro-level of analysis that 
measures engagement such as, hashtags or emotionalism expressed around each text. The study conducted by Kim, Ables and Yang (2012) examined how followers interacted with library-generated content, specifically identifying the individuals who retweet academic library messages. Their analysis consisted of 571 tweets from 10 institutions included in US News and World Reports" "Best Colleges and Universities" for 2012. Their findings revealed constituent units within the university, and students comprised the largest population retweeting library content comprising thirty percent of retweet activity. Shulman, Yep, \& Tome (2015) found similar results; their analysis of two academic libraries sought to identify "influential" followers in a library's network (179). Despite comprising less than ten percent of total followers, institutional accounts were the most powerful, meaning they not only retweeted the most, but also were vehicles through which tweets extended well beyond the libraries' network of students, faculty, and staff.

Stvilia and Gibradze (2014) explored factors that made academic library tweets "useful" - measuring both the number of retweets and rate of favorability. Data was collected using a Twitter API, resulting in 753 tweets, from six public university libraries in the United States. The study showed the most retweeted and favored content related to academic support services and library as place. A text mining approach guided AlDaihani and Abrahams' (2016) analysis. Data comprised 23,707 tweets, collected from 10 highly selective universities in the United States and United Kingdom. Analysis revealed the most common word frequencies in library tweets were "open", "special collections", and "save-the-date." Additionally, tweets relating to resources were the most common category of original tweet disseminated. Al-Daihani and Abrahams suggest text mining as a helpful tool for decision-making, marketing, and outreach.

\section{Methodology}

We measure engagement by examining a set of objective measures namely retweets and sentiment. These objective measures allowed us to produce a more reliable data set around user behaviours with the following research questions: RQ1: Are followers retweeting library content? RQ2: What sentiment is expressed around library generated Tweets? The study captured Twitter activity from seventeen HBCU's libraries,

over an eighteen-month period (December 2013-July 2015), using the IBM Watson Twitter Analytic Engine, following Suh et. al's model of data extraction and hashtag 
categorization (Suh, Hong, Pirolli, \& Chi, 2010); of the 100 four-year HBCUs we found 17 with active Twitter accounts (i.e. accounts with at least one tweet). Watson utilized the following variables to measure the aforementioned research questions: library followers and sentiment. Watson's harvesting approach was composed of the following three components: extracting data from the data provider (i.e. the Twitter data servers) via the Twitter application program interface (API); parsing, integrating, and storing the data in a NoSQL database that resided in IBM Watson. IBM Watson allowed the exploration of Twitter relationships between information producers and followers (i.e. libraries and followers). IBM Watson's analytic engine treated each library's Twitter username as a seed, which corresponded to followers, limiting each library's data set to 15,664 users having any connection to the library.

\section{Results and Discussion}

The dataset comprised 23,354 institutional tweets, 13,259 hashtags, 1,074 mentions, and 6,880 retweets. The first research question examined if followers retweeted library content. We found that the majority of followers did not propagate library content, which suggests that user engagement, in the context of a participatory library culture, was quite low. However, the analysis revealed that a large percentage of libraries did not disseminate library related tweets; rather they engaged in what we term institutional boosterism. This content related to sporting events, campus activities and popular culture news items. Del Bosque, Leif, and Skarl 2012, also found that libraries in their study "included tweets about campus and community events" (p. 210), however, the majority of library tweets reflected "library resources," "posting hours," and "library events" (p. 210).

Based on our analysis of retweets we observed only $11 \%$ of followers retweeted library content. Although, $60 \%$ of library-generated tweets propagated, this activity resulted from non-followers rather than entities directly connected to the libraries' Twitter accounts. This phenomenon is likely related to the exceedingly large number of non-library related hashtags observed in the data-set. A large percentage of hashtags, corresponded to institutional boosterism, allowing Twitter users with university ties a way to easily discover and subsequently retweet engaging, albeit non-library related content. This suggests, most libraries were engaged in a type of self-talk, rather than fostering a participatory culture of followers via social media. Sentiment characteristics 
in HBCU library tweets were found to range from a -2.5 to 2.5. We observed three distinctive clusters around engagement within this Twitter community; libraries at small private institutions, with enrollments under 1,500 students, expressed the most positive sentiment based on their scores, in contrast to much larger colleges and universities, which manifested as overall negative sentiment. Paradoxically, the cluster of libraries expressing the most positive sentiment, were also not tweeting about library related phenomena.

\section{Conclusion}

Our study found little evidence of two-way communication occurring within Twitter. Rather, libraries in this study appeared to use Twitter as a basic broadcasting service that promotes institutional boosterism. The boosterism-oriented content was frequently retweeted, garnered positive sentiment scores, and reached a wide audience of Twitter users. Based on our analysis, we were unable to discern any tangible benefit for HBCU academic libraries using Twitter in this manner. It would be advantageous if future information scientists produce research that can substantiate continued allocation of time and effort with regards to social media, as a tool for community engagement and outreach.

\section{References}

Al-Daihani, S. M., \& Abrahams, A. (2016). A Text Mining Analysis of Academic Libraries' Tweets. The Journal of Academic Librarianship, 42(2), 135-143.

Buschman, J. (2003). Dismantling the public sphere: situating and sustaining librarianship in the age of the new public philosophy. Westport, Conn.: Libraries Unlimited.

Del Bosque, D., Leif, S. A., \& Skarl, S. (2012). Libraries atwitter: Trends in academic library tweeting. Reference Services Review, 40(2), 199-213.

Deodato, J. (2014). The patron as producer: libraries, web 2.0, and participatory culture. Journal of Documentation, 70(5), 734-758.

Dickson, A., \& Holley, R. P. (2010). Social networking in academic libraries: the possibilities and the concerns. New library world, 111(11/12), 468-479.

Kim, H. M., Abels, E. G., \& Yang, C. C. (2012). Who disseminates academic library information on Twitter? Proceedings of the American Society for Information Science and Technology, 49(1), 1-4. 
Shulman, J., Yep, J., \& Tomé, D. (2015). Leveraging the Power of a Twitter Network for Library Promotion. The Journal of Academic Librarianship, 41(2), 178-185.

Stvilia, B., \& Gibradze, L. (2014). What do academic libraries tweet about, and what makes a library tweet useful? Library \& Information Science Research, 36(3), 136-141.

Suh, B., Hong, L., Pirolli, P., \& Chi, E. H. (2010). Want to be retweeted? large scale analytics on factors impacting retweet in twitter network. Paper presented at the Social computing (socialcom), 2010 ieee second international conference on. 